\title{
Cáncer de Ovario en el Hospital Universitario del Valle Período 1974 - 1984
}

\author{
Dres.: Jorge E. Zúñiga C.*ly Alba Lucía Mondragón**
}

No obstante los avances en la Oncología Ginecológica el Cáncer del Ovario continua siendo un desafío para el ginecólogo.

"No existen medios conocidos para la detección del Cáncer Ovárico antes de efectuar un examen físico en conjunto con Biopsia - Citología - Cirugía o las tres" (1).

La falta de buenos resultados se debe en gran parte al avanzado estado de la enfermedad cuando la mujer va a la consulta médica.

En los Estados Unidos de Norteamérica se desarrollará Cáncer de Ovario en 1 de cada 70 niñas recién nacidas;'y una mujer muere por la misma enfermedad cada 50 minutos aproximadamente.

\footnotetext{
* Profesor Asociado Univalle. Jefe Servicio de Ginecología. Hospital Universitario. Cali.

** Residente 3er. Año. Depto. Ginecoobstetricia. Hospital Universitario. Cali.
}

12 de cada 1.000 mujeres por encima de los 40 años sufrirán de este tumor y sólo 2 ó 3 pueden ser curadas (4).

Se considera que causa el $6 \%$ de todas las muertes por Neoplasia en la mujer.

Entre las causas de muerte por Cáncer del aparato genital femenino, ocupa el 4o. lugar. Es el proceso maligno que con mayor frecuencia causa la muerte en las mujeres.

En promedio el $60 \%$ de las mujeres acuden al médico con enfermedad en etapa avanzada (Estados Clínicos III y IV) de la clasificación de la Figo.

El 20 al 50\% de las mujeres después de operadas y recibir complemento con radioterapia mostrarán recaída y necesitarán tratamiento adicional (3).

Los tumores malignos del Ovario pueden ocurrir a cualquier edad, incluyendo la infancia y la adolescencia.

Los tumores de las Células Germinales son más comunes en las mujeres antes 
de los 20 años de edad; mientras que los tumores de origen epitelial son vistos en mujeres por encima de los 50 años de edad; con aumento hasta los 80 años. La gran mayoría de casos se observa en la década de los 50-59 años (4).

Parece tener una tendencia familiar; con alta incidencia en países desarrollados, lo que sugiere que algunos productos físicos y químicos de la industria podrían considerarse entre las principales causas de los tumores epiteliales.

Los mecanismos etiológicos involucrados en el Cáncer de Ovario son limitados a informaciones fragmentarias.

En nuestro medio el panorama de esta enfermedad permanece muy sombrío.

La mayoría de las pacientes llegan a la consulta médica en etapas ó estadios muy avanzados de la enfermedad. Por otro lado se tienen muchas dificultades para hacer un tratamiento óptimo ya sea por cirugía - radioterapia o quimioterapia; en cuanto a la primera acción se presentan problemas serios por falta de recursos dado el déficit presupuestal de los Hospitales como el nuestro. Para la Radioterapia no se cuenta con equipos modernos y los que hay ya están obsoletos y con una recarga de trabajo que los hace cada vez más deficientes.

En cuanto a la Quimioterapia se consiguen con muchas dificultades algunas drogas y en la mayoría de los casos los tratamientos son parciales y muy incompletos; el médico queda limitado a muy pocas acciones y hace lo que puede con los elementos que tiene en un momento dado.

Por lo anterior, los resultados obtenidos en el manejo de esta enfermedad son muy pobres y no pueden ser comparados con otros países donde se cuenta con mejores condiciones para afrontar este desafío.

\section{PACIENTES $Y$ METODOS}

Este estudio comprende el análisis de 154 casos de tumores malignos de Ovario tratados por nosotros en la década 1974-1984.

Se diseñó un protocolo para hacer el análisis retrospectivo de todos y cada uno de los casos teniendo en cuenta los siguientes parámetros.

Procedencia

Edad

Raza

Paridad

Síntomas

Diagnóstico

Estudio Histológicó

Estadio Clínico

Metastasis Tumorales

Tumores Malignos Asociados

Laparotomía de 2 a. mirada

Tratamiento inicial

Tratamiento después de la $2 \mathrm{a}$.

Laparotomía

Morbilidad Operatoria

Tiempo de Seguimiento

Tiempo de Recuperación

Recidivas

Tratamiento de Ias Recidivas

Se pudo obtener un promedio de 15 casos por año en la década estudiada.

\section{Edad}

Los límites de edad estuvieron entre los $21 / 2$ años y los 80 años. El mayor número de casos en la década de los 40 a los 50 años. 
Sólo 26 casos por debajo de los 30 años; y únicamente 4 casos después de los 70 años.

\section{Raza}

Como siempre ocurre en nuestro medio la mayoría de las pacientes eran mestizas. 60 casos para un $39 \%$ del grupo estudiado.

\section{Paridad}

También como es de común ocurrencia en nuestro medio la mayoría de las pacientes son grandes multíparas. 58 casos con 4 hijos para un $38 \%$ aproximadamente. Llama la atención el número de pacientes Nulíparas 33 casos para un $22 \%$ aproximadamente.

\section{ESTUDIO HISTOLOGICO}

El tumor más irecuente en este grupo fue el Carcinoma Seroso Papilar para un. total de 57 casos.

El segundo en frecuencia fue el tumor de la Granulosa para 20 casos.

Por el número de casos es importante hacer notar otros tumores así:

Carcinoma Mucinoso 13 casos Adenocarcinoma no Diferenciado 14 casos Carcinoma, endometrioide $\quad 11$ casos El tumor de senos endodermicos 7 casos El Disgerminoma 6 casos El Tumor de Krukemberg 6 casos

En los otros tipos de tumores la frecuencia de casos fue muy baja.

\section{CORRELACION DE LA EDAD CON EL TIPO HISTOLOGICO DEL TUMOR Y ESTADIO DE LA ENFERMEDAD}

Para el Grupo de Edad

$0-10$ años.

1 caso de Linfoma

El diagnóstico se hizo por la presencia de una masa tumoral en Ovario.

$20-30$ años Estadio I 8 casos

Tumor de Células de la Granulosa 2 casos

Adenocarcinoma Seroso Papilar 2 casos

Arreno-Blastoma

1 caso

Disgerminoma

1 caso

Tumor de Senos Endodérmicos 1 caso

Tecoma

1 caso

$20-30$ años Estadio II 2 casos

Disgerminoma 1 caso

Tumor de Krukemberg 1 caso

$10-20$ años Estadio III 3 casos

Tumor de Senos Endodérmicos 1 caso

Disgerminoma 1 caso

Coriocarcinoma Metastásico 1 caso

\section{EDAD* ESTADIO CLINICO Y TIPO HISTOLOGICO}

$20-30$ años Estadio III

Cistoadenocarcinoma Seroso 2 casos

$10-20$ años Estadio III

Disgerminoma 1 caso

Tumor de Senos Endodérmicos 1 caso

$0-10$ años Estadio IV

Linfoma 1 caso

$10-20$ años 2 casos 
Tumor de Células de la Granulosa 1 caso

Disgerminoma

1 caso

$$
20-30 \text { años }
$$

Cistoadenocarcinoma Seroso

2 casos

Disgerminoma

1 caso

Tumor Senos Endodérmicos

Mesotelioma Maligno

1 caso

1 caso

\section{EDAD - ESTADIO CLINICO - TIPO HISTOLOGICO. RESUMEN}

\begin{tabular}{|c|c|c|c|}
\hline \multicolumn{2}{|l|}{$0-10$ Años } & \multicolumn{2}{|c|}{1 caso Linfoma } \\
\hline $10-20$ & & $\begin{array}{l}7 \text { casos } \\
\text { Estados II }\end{array}$ & 3 casos \\
\hline T. Senos Endodérmicos & 2 & \multirow{4}{*}{$\begin{array}{l}\text { Estados III } \\
\text { Estados IV }\end{array}$} & 2 casos \\
\hline Disgerminoma & 3 & & 2 casos \\
\hline Coriocarcinoma & 1 & & \\
\hline T. Células Granulosa & 1 & & \\
\hline $20-30$ años & 17 casos & & \\
\hline Disgerminoma & 3 & 1 & 8 casos \\
\hline T. Senos END. & 2 & II & 2 casos \\
\hline T.C. La Granulosa & 2 & III & 2 casos \\
\hline Arrenoblastoma & 1 & IV & 5 casos \\
\hline Cistoadenocarcinoma Se & so Papilar & & 6 casos \\
\hline Tumor de Krukemberg & & & 1 caso \\
\hline Mesoteliomá & & & 1 caso \\
\hline Tecoma & & & 1 caso \\
\hline
\end{tabular}

\section{Procedencia de las Pacientes}

En 76 casos las pacientes procedían de la Ciudad de Cali (49\%).

En 58 casos las pacientes venían de otras ciudades del Valle (38\%).

En 20 casos las pacientes provenían fuera del Depto. del Valle (13\%).

\section{SINTOMAS MAS COMUNES}

El síntoma más común fue el dolor abdominal en 86 casos para un $56 \%$.
Siguió en importancia el aumento o crecimiento del abdomen en 66 casos para un $43 \%$.

La presencia de masa abdominal en 44 casos para un $28 \%$

Síntomas digestivos en 50 casos para un $32 \%$

Síntomas urinarios en 30 casos para un $19 \%$.

Pérdida de peso y anorexia 38 casos para un $25 \%$.

Hemorragia genital en 18 casos para $12 \%$.

\section{OTROS SINTOMAS MENORES}

Amenorrea -Síntomas Respiratorios. Equivocación con Embarazo.

Leucorreas.

Llama la atención el porcentaje de pacientes asintomáticas $2,6 \%$ Estas pacientes correspondían a tumores de origen epitelial la mayoría de casos.

\section{RESUMEN ANTERIOR}

Dolor Abdominal

Crecimiento del Abdomen

$8656 \%$

Síntomas Digestivos

$\begin{array}{lll}66 & 43 \quad \%\end{array}$

Presencia de Masa.

$\begin{array}{lll}50 & 32 & \circ\end{array}$

Pérdida de Peso - Anorexia $38 \quad 25$ \%

Síntomas Urinarios $\quad 3019$ \%

Hemorragia Genital $\quad 18 \quad 12 \%$

Amenorrea $21.2 \%$

Síntomas Respiratorios $\quad 2 \quad 1.2$

$\begin{array}{lll}\text { Leucorrea } & 1 & 0.6\end{array}$

Confusión con Embarazo $\quad 2 \quad 1.2$

\section{NOTA}

Como una misma paciente tiene o presenta varios síntomas la suma de 
pacientes por síntomas es mayor que el número de casos y lo mismo ocurre con los porcentajes.

\section{CASOS POR ESTADIOS CLINICOS}

$\begin{array}{llrrrr}\text { ESTADIO I } & \text { A.- } & 26 & \text { casos } & \\ & \text { B.- } & 2 & \text { casos } & \\ & \text { C. }- & \frac{5}{33} & \text { casos } & \\ & & & \text { casos } & 21 \% \\ \text { ESTADIO II } & \text { A. }- & 13 & \text { casos } & \\ & \text { B. }- & 2 & \text { casos } & \\ & \text { C. } & \frac{2}{17} & \text { casos } & \\ & & \text { casos } & 11 \% \\ \text { ESTADIO III } & & 48 & \text { casos } & 31 \% \\ \text { ESTADIO IV } & & 53 & \text { casos } & 34 \%\end{array}$

Sin dato en la H. Clínica

Llama la atención el porcentaje tan alto de casos avanzados para los Estados Clínicos III y IV.

Se tuvo un porcentaje del $65 \%$ lo cual es grave si se tiene en cuenta que sólo el $5 \%$ de estas pacientes llega a sobrevivir 5 años (2).

En la Etapa I. Se encontraron 33 casos que representan un $21 \%$ del total de casos: es en este estadio cuando las pacientes tienen mejor pronóstico por el porcentaje al to de recuperacion a 5 años.

\section{METODIOS DE DIAGNOSTICO}

En 128 casos se empleó como método de diagnóstico la Laparotomía y la toma de Biopsias; correspondientes al $83 \%$ de los casos.
En 12 casos se hizo el diagnóstico utilizando el estudio de Células Malignas por Paracentesis $8 \%$.

* En 12 casos el estudio de la metastasis orientó el diagnóstico 8 \%

Laparotomía y Biopsias $128 \quad 83 \quad \%$ Citología por Paracentesis $12 \quad 8 \%$ Estudio de la Metastasis $\quad 12 \quad 8 \%$ Sin dato en $\mathrm{H}$. Clínica $\quad 2 \quad 1 \%$

TOTAL $\overline{154} \overline{100} \%$

$\begin{array}{ll}\text { * Ganglio Inguinal } & 6 \text { casos } \\ \text { Ganglio Supraclavicular } & 3 \text { casos } \\ \text { Biopsia de Vejiga } & 1 \text { caso } \\ \text { Biopsia de Endometrio } & 1 \text { caso } \\ \text { Biopsia por Laparoscopia } & 1 \text { caso }\end{array}$
(Al efectuar Lig. de Trompas)

\section{EXTENSION DEL TUMOR}

$\begin{array}{lrr}\text { A. Organos Pélvicos } & 104 & 68 \% \\ \text { Diseminación Intra -- } & & \\ \text { Abdominal } & 103 & 67 \% \\ \text { Metastasis al Tórax } & 28 & 18 \% \\ \text { Metastasis Retroperitoneales } & 12 & 8 \% \\ \text { Otras Metastasis } & 13 & 8 \% \\ \text { Sin dato en la H.C. } & 3 & 2 \%\end{array}$

Otras Metastasis

Cerebro

Vagina

Riñón

Ganglio Inguinal

Cuello (Supraclavicular).

\section{DISCUSION}

El mecanismo primario de diseminación consiste en la penetración de la cápsula por el epitelio proliferativo y la implantación ulterior de las células clonigenas en las superficies paracólicas 
La diseminación intraperitoneal sigue las vías corrientes por las cuales el líquido peritoneal, los productos sépticos y particulas, son eliminados de la cavidad.

La vía principal desde la pelvis tiene dirección ascendente,siguiendo la acanaladura peritoneal derecha hasta la cara inferior del hemidiafragma derecho y de ahí, a una trama densa de linfáticos diafragmáticos.

Se ha apreciado ataque aislado del diafragma con tumores por lo demás localizados y la obstrucción de los linfáticos en el diafragma por émbolos tumorales, puede originar ascitis de comienzo temprano.

La proximidad de la pélvis con el epiplón, ciego, ileo terminal, y colon sigmoide hace que estos órganos sean sitios frecuentes de implantación temprana.

A menudo hay metastasis en ganglios para - aorticos pero la diseminación extra abdominal por esta ruta es lenta.

La diseminación hematogena suele ser manifestación tardia y en promedio en la mitad de mujeres que fallecen por esta. enfermedad, el tumor no se ha diseminado por fuera de la cavidad abdominal ni en los ganglios retroperitoneales.

En forma global, los carcinomas epiteliales por lo regular no son invasores, $y$ es poco frecuente que destruyan órganos vitales.

La obstrucción intestinal real, que no es tan común como suele pensarse, rara vez cede con la intervención quirúrgica. El efecto global de la interferencia en la función gastrointestinal, la producción de derrames serosos y las necesidades me- tabólicas de una masa cada vez mayor de tejido tumoral, conduce a la consunción progresiva.

En términos generales, lo que resta de vida a la mujer parece relacionarse con el volumen de tumor en proliferación, den. tro de la cavidad peritoneal (2-5).

La intervención terapéutica modifica muy poco el curso del cáncer ovárico en estado III excepto el brindar paliación por corto plazo. Desde hace muchos años se ha recomendado a los cirujanos extirpar la mayor cantidad posible del tumor en la operación inicial, pero la supervivencia ha mejorado únicamente si se ha eliminado todo. El tumor macroscópico o casi todo $(6-7)$.

De manera semejante, en promedio, la mitad de las mujeres tratadas por radiación o agentes de alquilación se benefician con la disminución del tumor a la mitad o todavía más, pero la prolongación importante de la vida obliga a eliminar todo el tumor apreciable (respuesta completa), una probabilidad de $20 \%$ o menos (8).

En el carcinoma de ovario en estado III el volumen del tumor sólido, en conjunto, suele exceder de un kilogramo de peso o un litro. Por lo expuesto, el volumen excede de $10^{12}$ células, número que representa 40 duplicaciones celulares, desde una sola célula neoplásica. El tiem. po de duplicación de los tumores voluminosos es lento, y refleja una baja fracción de proliferación y por esta razón, el lapso promedio de supervivencia de mujeres sin tratamiento es de siete meses a partir del diagnóstico.

De lo anterior, se aprecia el fracaso de la intervención quirúrgica, la radioterapia 
y la quimioterapia para prolongar la supervivencia, si el volumen del tumor sólo disminuye 50 \% .

El lograr una respuesta a la quimioterapia, esto es, la ausencia total de un tumor palpable después de la operación, ha dado por resultado lapsos de supervivencia promedio de 26 a 30 meses $(9-10)$ en estos casos, el volumen de células neoplásicas pudo haber disminuido a $1 \mathrm{~cm}$ cúbico $\left(10^{9}\right.$ células), lo cual representa una regresión de 99,9 por 100 del tamaño de la neoplasia, y una "Reversión" de $25 \%$ del lapso de proliferación tumoral. Sin duda estas estimaciones de volumen del tumor son burdas, pero de ellas se desprende que todo régimen terapéutico debe disminuir el volumen tumoral hasta llegar a no palpar la masa en más del $50 \%$ de las pacientes, antes que el efecto antineoplásico se refleje en una prolongación importante del promedio de una supervivencia, en meses.

\section{"SECOND LOOK" O SEGUNDA MIRADA}

Se realizó en 15 casos o sea el $10 \%$ de las pacientes obteniéndose los siguientes resultados.

En 10 casos o sea el $67 \%$ de este grupo no se encontró tumor residual, (casos libres de tumor).

En 5 casos se encontró tumor residual.

A este grupo de pacientes se les dio tratamiento así:

Quimioterapia en 5 casos

Radioterapia en 2 casos
Sólo sintomático en 8 casos.

(estados terminales).

En los casos con tumor residual los tipos Histológicos fueron:

Adenocarcinoma indeterminado 1 caso

Cistoadenocarcinoma Seroso 3 casos

Tumor células Granulosa 1 caso

Los estadios clínicos así:
II A - un caso
III tres casos
IV un caso

El T. de células de la Granulosa que se negó a tratamiento y regresu al año en estado IV.

Se utilizó como tratamiento Quimioterapia con agentes alquilantes como el melfalan y el tradicional esquema VAC.

Un caso recibió radioterapia.

Sobre vida 1 1/2 años en 4 casos

Los casos libres de tumor fueron:

\begin{tabular}{|c|c|}
\hline T. Endometroide & 2 casos \\
\hline Cistoadenocarcinoma Seroso & 5 casos \\
\hline Teratocarcinoma & 1 caso \\
\hline T. de Células Granulosa & 1 caso \\
\hline T. de Senos Endodérmicos & 1 caso \\
\hline TOTAL & asos \\
\hline
\end{tabular}

\section{ESTADIOS CLINICOS Y SOBRE VIDA DE LOS 10 CASOS NEGATIVOS \\ PARA TUMOR A LA SEGUNDA MIRADA}

E. $\quad$ IA 7 años neg.
IA $\quad$ Actualmente Neg. 

I A No volvió a control
I A A los 5 años Neg.
I A A los 9 años Neg.
II A Sobrevida $21 / 2$ años
III Sobrevida 3 años Sobrevida 3 años
Sobrevida 8 años
Sobrevida 3 años

Total 10 casos

Teniendo en cuenta los Estadios Clínicos la recuperación de estas pacientes fue muy buena.

\section{TUMORES MALIGNOS ASOCIADOS}

Se presentaron en 10 casos para un 6.3\% distribuidos así:

\begin{tabular}{|c|c|}
\hline En Mama & 3 casos \\
\hline En Cervix & 5 casos \\
\hline Otros & 2 casos \\
\hline TOTAL & $10 \mathrm{casos}$ \\
\hline
\end{tabular}

Estos casos se discriminan así:

Carcinoma de Cervix

In Situ 3 casos con adenocarcinoma mucinoso tecoma T.C. de la granulosa

CA. Cervix E II 1 caso (CA mucinoso) CA. Cervix E I 1 caso (CA Seroso Papilar) Tumor gástrico 1 caso Tumor de mama con CA. Endometroide CA. Seroso Papilar Cáncer de Vulva 1 caso.(en dos casos).

\section{MORBILIDAD OPERATORIA}

Se presentó en 18 casos para un $12 \%$. Infección en el 7\% de los casos (11 casos).

Trauma en Vías Urinarias en 5 casos $3 \%$.

Sangrado de Pediculo en 1 caso 0,6\%

Eventración en 1 caso $0,6 \%$.

\section{COMPLICACIONES POR QUIMIOTERAPIA}

Se presentaron en 21 pacientes así:

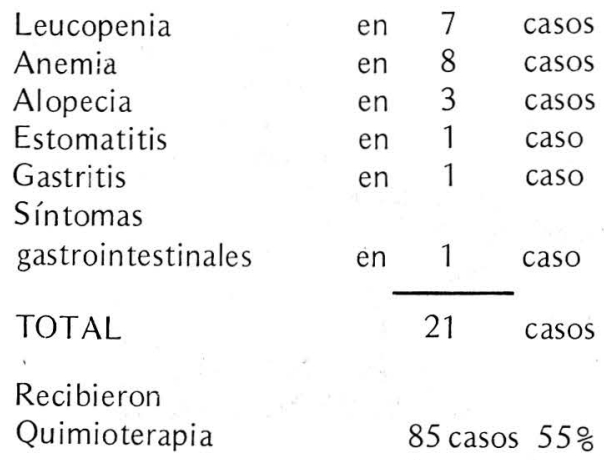

\section{COMPLICACIONES POR RADIOTERAPIA}

\begin{tabular}{|c|c|c|}
\hline Proctitis & 2 & casos \\
\hline Estenosis Rectal & 1 & caso \\
\hline Vagina Estenótica & 2 & casos \\
\hline Linfedema & 1 & caso \\
\hline Alt. de la Piel & 1 & caso \\
\hline $\begin{array}{l}\text { Pseudo - obstrucción } \\
\text { intestinal }\end{array}$ & 1 & caso \\
\hline TOTAL & 8 & casos \\
\hline
\end{tabular}

Recibieron Radioterapia 65 casos 42.\% 


\section{COMPLICACIONES POST-QUIRURGICAS}

Fístula entero vaginal - Vesico vaginal

Tenía CA de mama y CA Endometroide de Ovario.

En la 6a. década de la vida.

Tumor Estadio IV

Recibió Radioterapia. Previa Cirugía

(Anexo Histerectomía y Omen tectomía)

Complemento con Quimioterapia

No aceptó tratamiento de las Fistulas.

Fístula Vesico - Vaginal Post-Radioterapia

Paciente mayor de 70 años

Tumor Seroso Papilar E III

Se practicó Excisión Parcial del tumor

La fístula cerro en forma espontanea

Fístula Recto - Vaginal

Paciente en 5a. década de la vida

Tumor Seroso - Papilar

Estadio III

Anexo Histerectomía Total

Fístula Recto - Vaginal y/o Vesical

Paciente en 7a. década de la vida

Tumor Seroso Papilar E III

Anexo Histerectomía Sub Total

Fístula Entérica

Paciente en 7a. década de la vida

Tumor Seroso Papilar

Estado IV

Laparotomia y Biopsia

Fístula Urinaria con Hịdronefrosis y

Uremia
Tumor E II Endometroide

Edad 7a. década de la vida

Anex ohisterectomia

Lesión de Vejiga

Estenosis de Ureter

Fístula Entero - Vaginal (cierre

espontáneo)

Absceso de Cúpula Vaginal

Insuficiencia Renal Crónica

Paciente en 2a. a 3a. década de la vida

Laparotomia y Biopsia

Radioterapia

Anexohisterectomia Abd. Total

Omentectomia

Tumor Disgerminoma; viva a los 9 años.

Fístula Recto - Vaginal

Paciente en 5a. década de la vida

Tumor de Krukemberg E IV

Anexectomia Parcial

Colostomia Definitiva

Murió a los 10 meses

Otras Complicaciones

Insuficiencia Renal Crónica

Con insuficiencia cardiaca congestiva

Accidente - Cerebro - Vascular isquémico Aborto Espontáneo (Feto Muerto)

Trombo Embolismo Pulmonar

Insuficiencia Renal aguda con

Trombo Embolismo Pulmonar.

\section{RESUMEN}

Fístula Vesico - Vaginal 2

Fístula Recto - Vaginal 3

Fístula Enterica 1

Fístula Urinaria 1

Fístula Entero - Vaginal 1

TOTAL $\quad \overline{8} 5 \%$ 
RECIDIVAS

\begin{tabular}{l} 
En Estado I \\
\hline Recibieron Radioter \\
Quimioterapia \\
Cirugía \\
Recidiva Abdominal \\
Recidiva Pulmonar e \\
Recidiva Cerebro \\
T. de Células Claras \\
CA Embrionario
\end{tabular}

CA Indiferenciado

CA C. de la Granulosa

CA Mucinoso

$\begin{array}{lll}6 & \text { casos } & 18 \% \\ 2 & \text { casos }\end{array}$

3 casos

1 caso

$\begin{array}{ll}4 & \text { casos } \\ 1 & \text { caso } \\ 1 & \text { caso }\end{array}$

I A Anexohisterectomia Total

I B Cirugía Radioterapia Quimioterapia

I A Cirugía Radioterapia Quimioterapia

I A AnexectomíaQuimioterapia

I A Anexectomía.
Supraclaviculares

Cúpula Vaginal

1 caso

Recibieron Radioterapia 2 casos

Recibieron Quimioterapia 2 casos

Recibieron Cirugía

1 caso

CA Seroso Papilar

Cirugía -

Radioterapia -

Quimioterapia

2 años 2 meses sobre-vida

CA. Seroso Papilar

Cirugía -

Quimioterapia

Murió a los $91 / 2$ años

CA. Disgerminoma

Cirugía -

Radioterapia

Está viva a los 2 años 7 meses.

\section{TRATAMIENTO}

\section{RECIDIVAS}

\section{Estado II 2 Casos $11 \%$}

Recidiva en Vagina

Recidiva en Cervix y en

Hígado

1. CA. C. de la Granulosa II A Cirugía

Radioterapia

2. CA. Endometroide

1. Murió al año y 8 meses

2. A los 5 años Viva.

\section{RECIDIVAS}

\section{Estado III 4 casos $13 \%$}

Recidiva Abdominal

Recidiva Ganglios
2 casos

1 caso
IA. 6 Casos Anexohisterectomia

3 casos

Sobre-vida a más de 5 años

1 case Murió entre 2-3 años

1 caso No volvió a control

1 caso Murió por CA. de Vulva.

8 Casos Anexectomia

3 casos Sobre-vida a más de 5 años

1 caso Viva al momento del Estudio más de 1 año

1 caso Murió entre 1-2 años

3 casos No regresaron a Control

4 Casos Anexohisterectomia Total y Omentectomía

i caso Controlado 4 años No Volvió

3 casos Controlados 2-3 años no volvieron 

3 Casos
Anexectomia - Radioterapia
1 caso
A los 6 meses no volvió
2 casos

\section{Anexohisterectomia Total y Radioterapia}

2 casos

Control a más de 5 años

\section{Resección del Tumor}

1 caso

\author{
17 años bien \\ Recidiva a ese tiempo murió \\ al año
}

\section{Anexectomia y Quimioterapia}

1 caso Control 1 año bien

\section{Estadio I B}

Anexohisterectomia y Radioterapia

1 caso control a más de 5 años bien

Anexohisterectomia y Radioterapia con

Quimioterapia

1 caso murió en el curso del 3er. año

\section{Estadio I C}

Anexectomia 2 casos

1 caso viva bien a los 10 años

1 caso murió por anemia Drepanocitica Severa.

Anexohisterectomia 2 casos

Control hasta 6 meses

Anexectomia y Radioterapia 1 caso

Control hasta 6 meses.

\section{TRATAMIENTO}

\section{Estado II}

Anexectomia 1 caso

Control por $21 / 2$ años bien
Anexectomia y Radioterapia 1 Caso

Control por 5 años (murió por Recidiva)

Anexohisterectomia y Radioterapia 5 casos

2 casos control 5 años

1 caso control 3 años

1 caso control 2 años

1 caso control 3 años

Anexohisterectomia y Quimioterapia 2 casos No tuvieron control adecuado

Anexohisterectomia - Radioterapia y

Quimioterapia 3 casos

2 casos control a más de 5 años bien

1 caso 2 años y murió (Alteró el tratamiento).

Resección Parcial del Tumor y Quimioterapia 1 caso No regresó a control.

\section{Estado II B}

Anexohisterectomia y Radioterapia 1 caso Control bien por 5 años

Anexohisterectomia - Radioterapia y

Quimioterapia

1 caso control por 1 año

Estado II C

Anexohisterectomia - Quimioterapia Radioterapia 2 casos

1 caso murió a los 2 meses (T. Senos

Endodérmicos)

1 caso murió $11 / 2$ año. Por Tromboembolismo Pulmonar

\section{TRATAMIENTO}

\section{Estado III}

Anexectomia 1 caso no volvió a control

Anexectomia y Quimioterapia 3 casos

2 casos control por 6 meses murieron

1 caso control por 2 años murio 
Anexectomia y Radioterapia 5 casos

2 casos control 6 meses murieron

1 caso control 2 años vive

1 caso control 4 años vive

1. caso control a más de 5 años murio

Anexectomia - Radioterapia -

Quimioterapia 3 casos

2 casos control 2 años murieron

1 caso control 2 años no volvió

Anexohisterectomia Total y

Quimioterapia 6 casos

3 casos control 6 meses murieron

1 caso control 1 año murió

1 caso control más de 5 años

1 caso no regresó a control

Anexohisterectomia y Radioterapia

8 casos

1 caso 6 meses murió 2 casos más de 5 años

1 caso 2 años murió

2 casos 3 años no volvió 1 caso sin control

1 caso 4 años no volvió.

\section{TRATAMIENTO}

\section{E III}

Anexohisterectomia Total -

Radioterapia y Quimioterapia

8 casos

6 meses de control 1 caso murió

1 año de control 1 caso murió

2 años de control 2 casos murieron

3 años de control 1 caso vive

5 años de control 3 casos murieron.

Resección Parcial de la Masa y

Radioterapia y Quimioterapia

3 casos
1 caso con trol por 3 años murió

1 caso control por 2 años murió

1 caso control por 6 meses murió

Biopsia por Laparotomia - Radioterapia y Quimioterapia

Sólo Quimioterapia 5 casos

1 caso control por 1 año

2 casos control por 6 meses murieron

1 caso control por 1 1/2 años murió

1 caso control por 2 años murió

Radioterapia 1 caso

1 caso 6 meses murió

RADIOTERAPIA Y QUIMIOTERAPIA

1 caso control 15 años bien.

\section{TRATAMIENTO Y PRONOSTICO}

\section{ESTADOS IV}

AHAT con Omentectomia y Radioterapia 2 casos

1 caso recuperación por 6 meses

1 caso recuperación por 2 años

AHAT y Quimioterapia 6 casos

4 casos recuperación 6 meses

2 casos recuperación 1 año

AHAT. Quimioterapia y Radioterapia 1 caso

1 caso 3 años de recuperación

Resección Parcial del Tumor Radioterapia

y Quimioterapia

1 caso 3 años de recuperación

AHAT 3 casos

Recuperación 6 meses 2 casos

Recuperación 4 años 1 caso 
Quimioterapia 1 caso recuperación 2 años Anexectomia 1 caso recuperación 1 año

Anexectomia y Quimioterapia 9 casos

Recuperación por 6 meses 7 casos

Recuperación por 1 año 1 caso

Recuperación por 2 años 1 caso

Anexectomia y Radioterapia 1 caso

Recuperación por 4 años no regresó

Se trataba de Disgerminoma

Anexectomia - Radioterapia y

Quimioterapia

1 caso recuperación 1 año

Biopsia Sin Tratamiento 3 casos

Muerte en los primeros 6 meses

Biopsia y Radioterapia 1 caso

Recuperación 6 meses

Biopsia y Quimioterapia 18 casos

Recuperación hasta 6 meses 13 casos

Recuperación hasta 1 año 3 casos

Recuperación hasta 2 años 1 caso

Recuperación hasta 3 años 1 caso

Biopsia - Radioterapia y Quimioterapia

1 caso

Recuperación hasta 2 años.

Biopsia - AHAT - Radioterapia y

Quimioterapia 1 caso

Recuperación hasta 3 años.

Biopsia AHAT y Radioterapia

Recuperación 9 años

Tratamiento Sintomático 4 casos

Muerte primeros 6 meses
TOTAL

$0-6$ meses

$6-12$ meses

$1-2$ años

$2-3$ años

3 - 4 años

$4-5$ años

$>5$ años

57 Casos

Estado IV

35

$61 \%$

$14 \%$

$12 \%$

7 \%

$2 \%$

$2 \%$

$2 \%$

\section{DISTRIBUCION DE CASOS POR AÑOS}

\begin{tabular}{|r|r|}
\hline 1974 & 10 \\
1975 & 9 \\
1976 & 18 \\
1977 & 13 \\
1978 & 20 \\
1979 & 15 \\
1980 & 18 \\
1981 & 11 \\
1982 & 17 \\
1983 & 16 \\
1984 & 8 \\
\hline
\end{tabular}

$\bar{X}: 15$ Casos / Año

PROCEDENCIA

\begin{tabular}{|l|c|c|}
\cline { 2 - 3 } \multicolumn{1}{c|}{} & Casos & $\%$ \\
\hline Cali & 76 & 49 \\
Valle & 58 & 38 \\
Otros Deptos & 20 & 13 \\
\hline
\end{tabular}


PARIDAD

\begin{tabular}{|c|c|r|}
\hline Ne Partos & Casos & $\%$ \\
\hline 1 & 23 & 15.0 \\
2 & 17 & 11.0 \\
3 & 13 & 8.4 \\
4 & 58 & 37.6 \\
Nuliparas & 33 & 21.4 \\
Sin dato & 10 & 6.6 \\
\hline
\end{tabular}

R A Z A

\begin{tabular}{|l|c|c|}
\cline { 2 - 3 } \multicolumn{1}{c|}{} & Casos & $\%$ \\
\hline Mestiza & 60 & 39.0 \\
\hline Negra & 17 & 11.0 \\
India & 1 & - \\
Sin dato & 58 & 38.0 \\
\hline
\end{tabular}

EDA D

\begin{tabular}{rrr}
$0-9$ & 2 & 1.0 \\
$10-19$ & 7 & 4.0 \\
$20-29$ & 17 & 11.0 \\
$30-39$ & 18 & 12.0 \\
\hline $40-49$ & 43 & 28.0 \\
\hline $50-59$ & 30 & 20.0 \\
$50-70$ & 33 & 21.0 \\
$>70$ & 4 & 3.0
\end{tabular}

Menor edad $21 / 2$ años

Meyor edad 85 años
TIPO HISTOLOGICO DEL TUMOR

\begin{tabular}{|l|r|r|}
\cline { 2 - 3 } \multicolumn{1}{c|}{} & Casos & $\%$ \\
\hline Cistoudenocarcinoma seroso & 57 & 37.0 \\
T. células de la granulosa & 20 & 13.0 \\
Adenocarcinoma no diferenciado & 14 & 9.0 \\
Cistoadenocarcinoma mucinoso & 13 & 8.0 \\
Cáncer endometroide & 11 & 7.0 \\
Tumor de senos endodermicos & 7 & 5.0 \\
Disgerminoma & 6 & 4.0 \\
Tumor de Krukemberg & 6 & 4.0 \\
Cáncer indiferenciado & 5 & 3.0 \\
Mesotelioma maligno & 2 & 1.0 \\
Teratocarcinoma & 2 & 1.0 \\
Tumor mixto & 2 & 1.0 \\
\hline
\end{tabular}

... tipo histológico del tumor

\begin{tabular}{|l|c|c|}
\cline { 2 - 3 } \multicolumn{1}{c|}{} & Casos & $\%$ \\
\hline Cáncer originado en teratoma & 1 & 0.7 \\
Carcinoide & 1 & 0.7 \\
Cáncer células claras & 1 & 0.7 \\
Coriocarcinoma metastásico & 1 & 0.7 \\
Cáncer embrionario & 1 & 0.7 \\
Tecoma & 1 & 0.7 \\
Cáncer anaplásico & 1 & 0.7 \\
Arrenoblastoma & 1 & 0.7 \\
Iinfona & 1 & 0.7 \\
\hline
\end{tabular}

Tin solo caso de tumor funcionante

SINTOMAS

\begin{tabular}{|l|c|c|}
\cline { 2 - 3 } \multicolumn{1}{l|}{} & CASOS & $\%$ \\
\hline $\begin{array}{l}\text { Crecimiento del } \\
\text { abdomen }\end{array}$ & 36 & 56.0 \\
$\begin{array}{l}\text { Sintomas } \\
\text { digestivos }\end{array}$ & 50 & 32.0 \\
$\begin{array}{l}\text { Sintomas } \\
\text { Urinarios }\end{array}$ & 30 & 19.0 \\
$\begin{array}{l}\text { Palpación masa } \\
\text { Cx el paciente }\end{array}$ & 44 & 28.0 \\
Pérdida de peso & 38 & 25.0 \\
$\begin{array}{l}\text { Hemorragia } \\
\text { genital }\end{array}$ & 18 & 19.0 \\
\hline
\end{tabular}


OTROS (Muy bajo porcentaje)

A menorrea

S. Respiratorios

Embarazo

Leucorrea

Anorexia

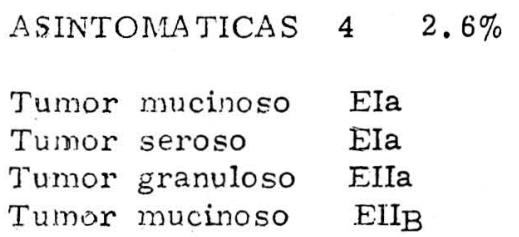

ESTADIOS CLINICOS

(Clasificación de la Figo)

\begin{tabular}{lrrrrr}
\hline E $_{\mathrm{I}}$ & $\mathrm{A}$ & 26 & & \\
& $\mathrm{~B}$ & 2 & 33 & $21 \%$ \\
& $\mathrm{C}$ & 5 & & & \\
\hline EII & $\mathrm{A}$ & 13 & & & \\
& $\mathrm{~B}$ & 2 & 17 & $11 \%$ \\
& $\mathrm{C}$ & 2 & & & \\
\hline EIII & & 48 & $31 \%$ \\
E IV & & 53 & $34 \%$ \\
\hline Sin dato & & 3 & $3 \%$ \\
\hline TOTAL & & & \\
\hline
\end{tabular}

METODOS DIAGNOSTICOS

\begin{tabular}{|l|r|r|}
\cline { 2 - 3 } \multicolumn{1}{c|}{} & Casos & \multicolumn{1}{c|}{$\%$} \\
\hline Laparotomáa y Biopsia & 128 & 83.0 \\
Por citologia L. Ascitico & 12 & 8.0 \\
Biopsia de la metástasis & 12 & 8.0 \\
Sin dato & 2 & 1.0 \\
\hline TOTAL & 154 & 100.0 \\
\hline
\end{tabular}

EXTENSION DEL TUMOR

\begin{tabular}{|l|r|r|}
\cline { 2 - 3 } \multicolumn{1}{c|}{} & Casos & $\%$ \\
\hline A Srganos pélvicos & 104 & 68.0 \\
A organos intraabdominales & 103 & 67.0 \\
Al torax & 28 & 18.0 \\
Al retroperitoneo & 12 & 8.0 \\
* Otros & 13 & 8.0 \\
Sin dato & 3 & 2.0 \\
\hline * Cerebro & & \\
Vagina & & \\
Ganglio inguinal & & \\
Ganglio cervical & \\
Rinón & \\
\hline
\end{tabular}

\begin{tabular}{|c|c|}
\hline $\begin{aligned} & \text { "SECOND - LOOK" SEGUNDA } 15 \text { Casos } 10 \% \\
&\end{aligned}$ & MIRADA \\
\hline $\begin{array}{l}\text { Negativos para tumor } \\
\text { Positivo para tumor } \\
\text { intra-abdominal }\end{array}$ & 10 Casos \\
\hline
\end{tabular}

TUMORES MALIGNOS ASOCLADOS

\begin{tabular}{lll}
\hline \multicolumn{1}{c}{10 Casos } & $6.5 \%$ \\
\hline Mama & 3 \\
Cervix & 5 \\
Gástẹ ico & 1. \\
Vulva & 1 \\
\hline
\end{tabular}

\begin{tabular}{lcc} 
MORBILIDAD & OPERATORIA \\
\hline & 18 & $12.0 \%$ \\
\hline $\begin{array}{l}\text { Infeccion urinaria } \\
\text { Trauma vas uri- }\end{array}$ & 11 & 7 \\
narias & 5 & 3 \\
Sangrado de pedículo & 1 & 0.6 \\
Eventración & 1 & 0.6 \\
\hline
\end{tabular}

REACCIONES POR QUIMIOTERA PLA

\begin{tabular}{lc}
\hline & 20 \\
\hline & Casos \\
Leucopenia & 7 \\
Anemia & 8 \\
Alopecia & 3 \\
Estomatitis & 1 \\
Gastritis & 1 \\
\hline Recibieron & quimioterapia 85 casos \\
$55 \%$ &
\end{tabular}


REACCIONES POR RADIOTERA PLA 8 Casos

\begin{tabular}{lc}
8 Casos & Casos \\
\hline & 2 \\
Proctitis & 1 \\
Estenosis rectal & 2 \\
Vagina estenotica & 1 \\
Alt. de la piel & 1 \\
Obstruccion intest. & 1 \\
\hline Recibieron radioterapia & 65 Casos \\
$42 \%$ &
\end{tabular}

\section{COMPLICA CIONES POST-QUIRURGICAS}

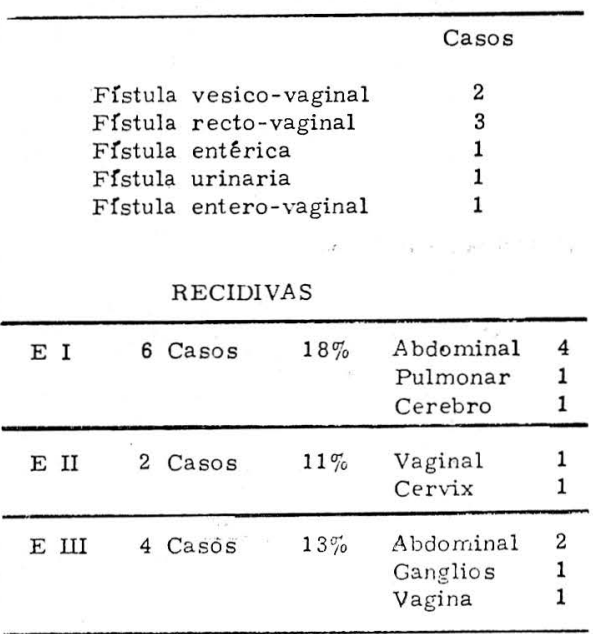

TRATA MIENTO

CASOS

\begin{tabular}{|c|c|c|}
\hline I A. & $\begin{array}{l}\text { Anexohisterectomía } \\
\text { Anexectomía } \\
\text { Anexohisterectomfa y omentectomía P. } \\
\text { Anexectomía y radioterapia } \\
\text { Anexohisterectomía y radioterapia } \\
\text { Resección del tumor } \\
\text { Anexectomfa y quimioterapia }\end{array}$ & $\begin{array}{l}6 \\
8 \\
4 \\
3 \\
2 \\
1 \\
1\end{array}$ \\
\hline I $\mathrm{B}$. & $\begin{array}{l}\text { Anexohisterectomfa y radioterapia } \\
\text { Anexohisterectomfa y quimioterapia }\end{array}$ & $\begin{array}{l}1 \\
1\end{array}$ \\
\hline I C. & $\begin{array}{l}\text { Anexectomfa } \\
\text { Anexohisterectomía } \\
\text { Anexectomfa y radioterapia }\end{array}$ & $\begin{array}{l}2 \\
2 \\
1\end{array}$ \\
\hline
\end{tabular}

TRATA MIENTO

\begin{tabular}{|c|c|c|}
\hline & & CASOS \\
\hline \multirow[t]{6}{*}{ E II A. } & Anexectomia & 1 \\
\hline & Anexectomfa y radioterapia & 1 \\
\hline & Anexohisterectomia y radioterapia & 5 \\
\hline & Anexohisterectomía y quimioterapia & 2 \\
\hline & Anexohisterectomía Radiot. y Quimiot. & 3 \\
\hline & Resección P. del tumor y Quimiot. & 1 \\
\hline \multirow[t]{2}{*}{$\mathrm{E}$ II $\mathrm{B}$. } & Anexohisterectomía y radioterapia & 1 \\
\hline & $\begin{array}{l}\text { Anexohisterectomfa y radioterapia y } \\
\text { Quimioterapia }\end{array}$ & 1 \\
\hline \multirow[t]{3}{*}{$\mathrm{E}$ II C. } & A nexohisterectomfa & \\
\hline & Quimioterapia y radioterapia & 2 \\
\hline & TOTAL DE CASOS & 17 \\
\hline
\end{tabular}
$35 \%$

TRATA MLENTO

\begin{tabular}{lc}
\hline & CASOS \\
\hline E III. Anexectomía & 1 \\
Anexectomía y quimiot. & 3 \\
Anexectomía y radiot. & 5 \\
Anexectomía y radiot. con quimiot. & 3 \\
Anexohisterectomía y quimioterapia & 6 \\
Anexohisterectomía y radioterapia & 8 \\
Anexohisterectomía radiot. y quimiot. & 8 \\
Resección parcial tumor radiot y & 3 \\
quimiot. & 5 \\
Quimioterapia & 1 \\
Radioterapia & 1 \\
Radiot. y quimiot. & 44 \\
\hline TOTAL DE CASOS & \\
\hline Recuperación a 5 anos o más 4 casos & \\
O\% & \\
\hline
\end{tabular}

E IV. AHAT y Radiot.

AHAT Y Quimiot.

$\triangle H A T$ Radioterapia y Quimiot.

Resección parcial radiot. y quimiot.

AHAT

Quimioterapia

Anexectomía

Anexectomía y quimioterapia

Anexectomía y radioterapia

Anexectomfa radioterapia y quimiot.

Biopsia y radioterapia

Biopsia y quimioterapia

Biopsia radiot. y quimioterapia

Sintomático

TOTAL DE CASOS

5

Recuperación a 5 anos o más 2 Casos $4 \%$ 


\section{PROTOCOLO PARA ESTUDIO DE LOS CANCER DE}

OVARIO DEL H.U.V.PERIODO 1974 - 1984

TRABAJO DESCRIPTIVO

H. C.

NOMBRE

FECHA DE DIAGNOSTICO

PROCEDENCIA: 1. CALI

2. VALLE

RAZA: 1. NEGRA

3. OTROS

2. BLANCA

3. MESTIZA

4. SIN DATO

EDAD:

$\begin{array}{ll}\text { 1. } & 0-10 \\ \text { 2. } & 10-20 \\ \text { 3. } & 20-30 \\ \text { 4. } & 30-40 \\ \text { 5. } & 40-50 \\ \text { 6. } & 50-60 \\ \text { 7. } & 60-70 \\ \text { 8. } & \text { Mayor } 70\end{array}$

PARIDAD:

1. 1 hijo

2. 2 hijos

3. 3 hijos

4. 4 hijos o más

5. Ningún hijo

6. Sin dato

SINTOMAS:
1. Dolor
2. Sensación de masa
3. Distención abdominal
4. Hemorragia vaginal
5. Síntomas digestivos
6. Síntomas urinarios
7. Ninguno

DIAGNOSTICO:

$\begin{array}{ll}\text { 1. } & \text { Clínico } \\ \text { 2. } & \text { Paracentesis } \\ \text { 3. } & \text { Laparotomia } \\ \text { 4. } & \text { Ecografıa } \\ \text { 5. } & \text { Biopsia }\end{array}$

DIAGNOSTICO HISTOPATOLOGICO

ESTADIO CLINICO AL Dx. Qx.

1. EI Ia

$\mathrm{Ib}$

Ic

2. EII

IIa

IIb

IIC
TUMORES MALIGNOS ASOCIADOS

1. Ca. mama

2. Ca. de cervix

3. Ca. endometrio

4. Otros

5. Ninguno

6. Sin dato

3. EIII

(Continúa) 


\section{(Continuación)}

EXTENSION DEL TUMOR

1. Organos pélvicos

2. Intraabdominal

3. Tórax

4. Retroperitoneal

5. Otros
LAPAROTOMIA SEGUNDA MIRADA

1. $\quad \mathrm{Si}$
$2 . \quad$ No
RESULTADO LAPAROTOMIA SEGUNDA
$\begin{array}{ll}\text { 1 } & \text { Libre de tumor } \\ \text { 2. } & \text { Compromiso tumoral }\end{array}$

\section{TRATAMIENTO}

1. Anexectomia

2. Anexo-histerectomia abdominal total más omentectomia

3. Resección parcial de la masa

4. Biopsia, sin resección por ser técnicamente imposible

5. Quimioterapia:

Alteran CICLOS VAC, CICLOS

6. Radioterapia

7. Sintomática

TRATAMIENTO POST-LAPAROTOMIA SEGUNDA MIRADA
1. Quimioterapia
2. Radioterapia
3. Ninguno
4. Sintomático

MORBILIDAD OPERATORIA:

OTRAS COMPLICACIONES:
1. Ninguna
2. Infección
3. Vías urinarias
4. Sin dato

TIEMPO DE SEGUIMIENTO:

TIEMPO DE RECUPERACION:
1. $0-6$ meses
2. 7-12 meses
3. 1-2 años
4. $2-3$ años
5. 3-4 años
6. 4-5 años
7. 5-10 años
8 Mayor 10 años

(supervivencia)
1. $0-6$ meses
2. 7-12 meses
3. 1-2 años
4. 2-3 años
5. $3-4$ años
6. 4-5 años
7. 5-10 años
8. Mayor 10 años

RECIDIVA

TRATAMIENTO POST-RECIDIVAS

1. Abdominal
2. Pulmonar
3. Ganglios Supreclaviculares

1. Radioterapia

2. Quimioterapia

3. Sintomático 


\section{CONCLUSIONES}

Se presenta un estudio de 154 casos de Cáncer de Ovario en la última década en el Hospital Universitario del Valle que da un promedio de 15 casos por año.

El tumor más frecuente fue el Cistoadenocarcinoma Seroso Papilar con 57 casos ............

En el $49 \%$ de los casos las pacientes procedían de Cali y en el $38 \%$ venían del resto del Departamento del Valle.

Los cuatro síntomas más sobresalientes fueron en su orden dolor - crecimiento del abdomen - síntomas digestivos y pérdida de peso.

En el estadio I se encontraban el $21 \%$ de los pacientes en los Estadios III y IV estaban el $65 \%$ de las pacientes lo que reduce al mínimo las posibilidades de recuperación.

La laparotomia y la toma de Biopsia fue el principal método para comprobar el diagnóstico.

\section{REFERENCIAS}

1. Gynecol-Oncol. 13: 203-212, 1982.

2. GRIFFITHS C. T; FULLER A.F.Tratamiento Quirúrgico y Quimioterápico intensivo del Cáncer Ovárico Avanzado. Clínicas Quirúrgicas de Norte América. 1: 133-144, 1978.
La extensión del tumor se hizo primordialmente a la cavidad abdominal. En el $18 \%$ se encontraron metastasis al tórax.

En el $10 \%$ de los casos se practicó Laparotomia de 2a. mirada; en el 66\% de estos casos no se encontró tumor residual. En estos casos los tumores habían sido así: Adenocarcinoma Seroso Papilar 5 casos - y Endometrioide 2 casos - Teratocarcinoma 1 caso T. de C de la Granulosa 1 caso y T. de S. Endodérmicos 1 caso.

En el 6,5\% se asociaron otros tumores Malignos así: de Cervix en 5 casos; de Mama en 3 casos - CA Gástrico 1 caso y CA de Vulva en 1 caso.

Las recidivas en el Estadio I fueron del $18 \%$ para el grupo en cavidad abdominal 4 casos - en Pulmones un caso y en Cerebro 1 caso.

Las recidivas en el Estadio II fueron del $11 \%$ para el grupo una en vagina otra en Cervix.

Las recidivas en el Estadio III fueron del 13\% para el grupo así: En abdomen 2 casos - en ganglios periféricos 1 caso en Cúpula Vaginal 1 caso.

3. FISHER, R.I. and YOUNG R. C. Advances in the staging and treatment of ovarian cancer. Cancer, 39: 967-972., 1977.

4. DISAIA P: CREASMAN W. Clinical Gynecolgic Oncology The C.V. Mosby Company 1981. 
5. FELDMAN, G.B. and KNAPP, R.C. Limphatic Drainage of the Peritoneal Cavity and Its Significance in ovarian cancer. Am. J. Obstet. Gynecol 119: 991-994. 1974.

6. AURE, J.C.: HOEG, K. and KOLSTAD, P. Clinical and Histologic studies of Ovarian Carcinoma Obstet. Gynec, 37: 1-9. 1971.

7. GRIFFITHS, C.T.: Surgical Resection of Tumor Bulk in the Primary Treatment of Ovarian Carcinoma Nat'l cancer inst. monogi. 42: Symposium on Ovarian Cancer Washington, D.C.U.S Government Printing Office, 1975.
8. TOBIAS J.S.: and GRIFFITHS C.T Management of Ovarian Carcinoma $\mathrm{Cu}$ rrent Concepts and future prospects $\mathrm{N}$. Eng. I. Med. 294: $818-823$ and 877 882, 1976.

9. GRIFFITHS, C.T. GROGAN R.H. and HALL T.C. Advanced Ovarian Cancer Primary Treatment With Surgery Radioterapy and Chemotherapy Cancer 29:: 1-7, 1972.

10. YOUNG. R.C. CANELLOS S.P. CHABNER B.A. Chemotherapy of Advanced ovarian Carcinoma. A prospective Randomized Comparison of Phenylolamine mustard and High dose cyclophosphamide. Ginec. Oncol 2: 489-497. 1974. 